\title{
FAVORED ZODIAC FOR CELEBRITY BIRTHS
}

\author{
${ }^{1}$ Miah M. Adel and ${ }^{2}$ Saiyeeda F. Hossain and Hannah Johnson \\ ${ }^{1}$ Center for Interdisciplinary Sciences, Department of Chemistry and Physics, \\ University of Arkansas at Pine Bluff, Pine Bluff, AR 71601, United States \\ ${ }^{2}$ White Hall High School, 700 Bulldogs Drive, White Hall, AR 71602, United States
}

Received 2013-04-01; Revised 2013-09-16; Accepted 2014-02-13

\begin{abstract}
To find any favored zodiac sign for celebrity births, a sample of 100 celebrities were randomly selected from people of different walks of life. The sample contained politicians, natural scientists, social scientists, authors of literary works, social workers, humanitarian workers, business personnel, sports icons, singers, actors, actresses, etc. etc. from history and from the current time. The zodiac signs for the celebrities were found from their known dates of births. In the analysis of data, zodiac signs and the number of celebrities were represented as the independent $\mathrm{x}$ and the dependent $\mathrm{y}$ variables, respectively. For academic interests for the 9th grade high school juniors (at the time of the project performance), the co-authors of this article, as well as for the potentially illustrative uses in high school mathematics textbooks, bar and scatter plots were made, the line of best-fit and the equation of the line were found, probabilities of occurrences of celebrities for each of the zodiac signs were calculated and the correlation coefficients between the variables were determined for the sample. It was found that the zodiac Aquarius has the largest number of celebrities in the sample and that the two variables are moderately correlated. The sample sizes which were increased to 200 and then 300 . By including another 100 more celebrities to find if the trend remains unchanged. In all the three cases, Aquarius turned out to be the zodiac when most of the celebrities are born.
\end{abstract}

Keywords: Bar Plots, Celebrities, Zodiacs, Scatter Plots, Line of Best-Fit, Probability, Correlation Coefficient

\section{INTRODUCTION}

\subsection{Apparent Motion of the Zodiacs}

Figure 1 shows the sun at the center of the two concentric circles. The inner circle is the orbit of the earth around the sun. The outer circle is drawn through groups of stars called zodiac signs in the sky. In the sky, these groups of stars look about $8^{\circ} \mathrm{C}$ wide in the updown and $30^{\circ} \mathrm{C}$ long in the left-right directions. During the day time, stars are not visible because of the dazzling light from the sun. The inner circle is marked with three positions $\mathrm{A}, \mathrm{B}$ and $\mathrm{C}$ of the planet earth. Also, the outer circle is marked with the corresponding points $\mathrm{a}, \mathrm{b}$ and $\mathrm{c}$.
When the earth is at position A, the sun would be seen at the position a among the stars there if they were visible. When the earth is at position $B$, the sun would be seen among the group of stars at $b$ if the stars were visible. Similar things would happen for the positions $\mathrm{C}$ and c. It is observed that the background stars of the sun are changing which gives the impression that the sun moves around the planet earth. For the planet earth, because of its uniform motion (about 18.5 miles per second) around the sun, it seems stationary. Had there been any change of direction or an increase or a decrease in the motion, its stationary condition would be lost.

Corresponding Author: Miah M. Adel, Center for Interdisciplinary Sciences, Department of Chemistry and Physics, University of Arkansas at Pine Bluff, Pine Bluff, AR 71601, United States 


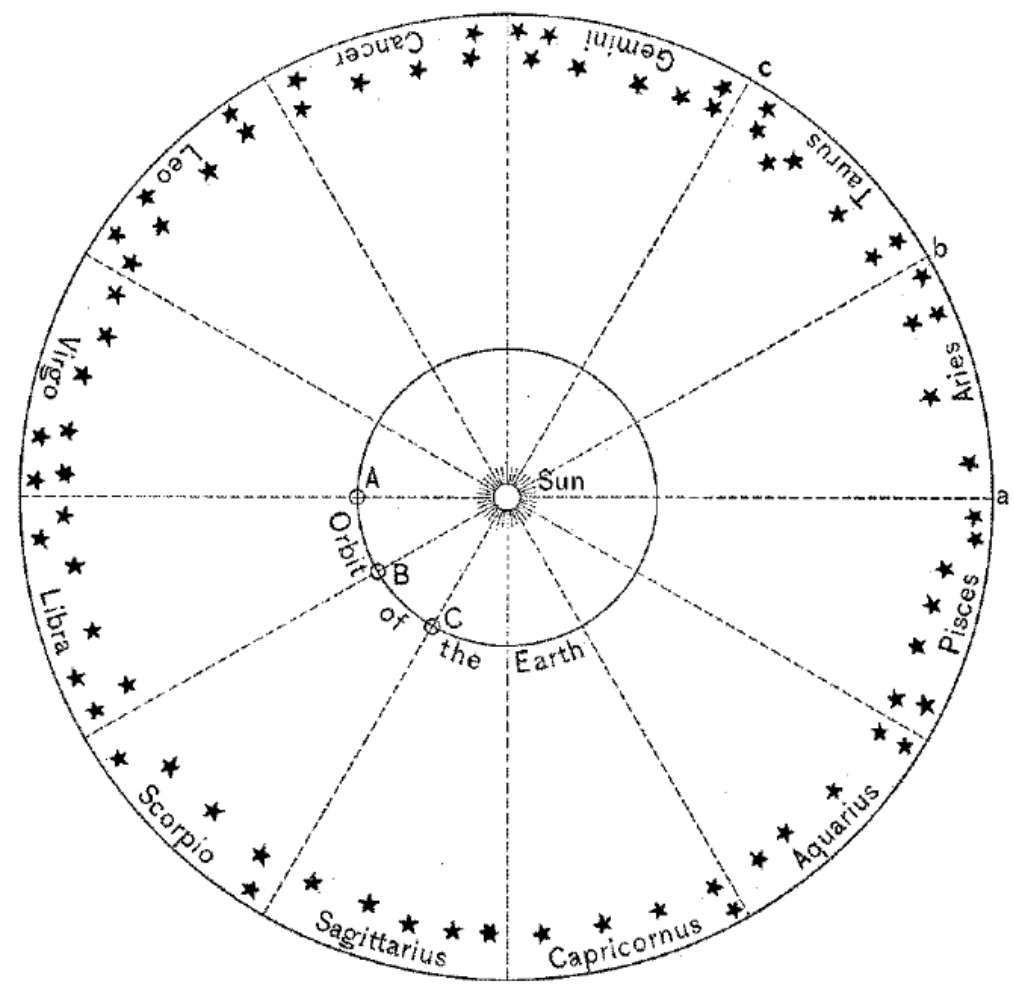

Fig. 1. Illustrates the apparent motion of the sun around us (http://www.astrology.com.au/astrology/12-signs-of-thezodiac/www.wikepedia.org)

\subsection{Zodiacs and Other Groups of Stars}

Zodiac means creatures which refer to the patterns of groups of stars in the clear night sky. There are 12 zodiac signs. The zodiac belt is the great circle (a circle over a sphere having the same center as the sphere) in which the sun apparently moves (http://www.astrology.com.au/astrology/12-signs-of-thezodiac/www.wikepedia.org) as illustrated above. Only the groups of stars behind the sun are called the zodiacs or the zodiac signs. There are 88 groups of stars including the zodiacs in the entire sky.

\subsection{Solar Crossings of the Zodiacs}

The sun enters a zodiac sign about the 21 st day of each month. The zodiac constellations are so named because the sun passes through them at a regular time annually (one sign per month). The names of the signs and the months when the sun enters each zodiac along with its brief traits are given in Table 1 below (www.psychicguild.com/).

\subsection{Zodiac Influence Testing for the Celebrities}

It is almost a part of universal culture to check one's fate with his/her zodiac sign. People feel quite comfortable with the prediction of his/her zodiac. While there is no scientific proof for the zodiac influences, no proof can be established because of the privacy of personal information, particularly, the date of birth of an individual. The dates of births and so the zodiacs of celebrities are known to the public. Their data could be studied to see if all or most of them are born in a particular zodiac and this is the focus of this article.

\subsection{Meaning, Duration and Traits pf Individual Zodiacs}

Table 1 records the meaning of each of the zodiac signs in column 1, the duration of each of the zodiac signs in column 2 and the brief traits of the individual zodiacs in column 3. 
Table 1: Zodiac signs' meanings, durations and traits

\begin{tabular}{lll}
\hline Latin name/English name & Time & Brief nature of celebrities \\
\hline Aries/the ram & Mar 21-Apr 20 & adventurous, energetic and courageous \\
Taurus/the bull & Apr 21-May 21 & patient, reliable and warmhearted \\
Gemini/the twins & May 22-Jun 21 & intellectual, youthful and lively \\
Cancer/the crab & Jun 22-Jul 22 & emotional, loving and cautious \\
Leo/the lion & Jul 23-Aug 21 & generous, creative and faithful \\
Virgo/the virgin & Aug 22-Sep 23 & reliable, practical and intelligent \\
Libra/the scales & Sep 24-Oct 23 & easygoing, charming and sociable \\
Scorpio/the scorpion & Oct 24-Nov 22 & determined, intuitive and passionate \\
Sagittarius/the archer & Nov 23-Dec 22 & optimistic, honest and intellectual \\
Capricorn/the goat & Dec 23-Jan 20 & practical, ambitious and patient \\
Aquarius/the water carrier & Jan 21-Feb 19 & friendly, loyal and original \\
Pisces/the fishes & Feb 20-Mar 20 & compassionate, intuitive and selfless \\
\hline
\end{tabular}

\section{MATERIALS AND METHODS}

\subsection{Definition of Celebrities and Their Random Selection}

Whoever has got a nationally and/or internationally positive publicity and has earned name and fame in his/her profession in history or in the current days' electronic and/or print media and whose personal information is available to the public was taken to be a celebrity. These celebrities appear in the worlds of politics, natural and social sciences, social and humanitarian services, literature, sports and athletics, recreational productions, arts and sculpture, etc. etc. The randomness and impartiality in the selection of the celebrity samples were maintained by picking the names of the celebrities first and then finding the dates of births and the zodiacs and not the other way around. The picking of the names of the top ones avoided the entire or partial selection in a regular form from a group of celebrities falling in a particular profession.

\subsection{Celebrity Data Spread}

About $40 \%$ of the celebrities were from the dead ones and $60 \%$ from the living ones. About $70 \%$ of them were males and $30 \%$ females. Less than $5 \%$ were Mexicans and Asians. No grouping was made based on ages because no age records were made as to when an individual became a celebrity.

\subsection{Data Analysis}

First, a random list of 100 celebrities from different walks of life was made. Their dates of births were found from wikepedia.com and found their zodiacs. The number of celebrities falling in each zodiac was counted. For the academic interests of the $9^{\text {th }}$-grade coauthors when they performed the project, bar plots of the zodiac signs Vs the number of celebrities were made and probabilities for the celebrities falling in a zodiac sign were calculated. Scatter plots were made with the zodiacs as the $\mathrm{x}$ variable and the number of celebrities as the $y$ variables. The line of best-fit using Microsoft Excel 2010 was found. The equation of the lines of best-fit were determined. The correlation coefficients (http://www.statisticshowto.com/articles/how-tocompute-pearsons-correlation-coefficients/) were determined to find how strongly the zodiac signs and the number of celebrities in them are related. The above procedure was repeated for sample sizes of 200 and then for 300 to find any continued feature common in all the sample sizes. Table 2 enlists the probabilities for the three sets of samples. Table 3 to 5 record the calculations for the correlation coefficients for sample sizes of 100, 200 and 300, respectively. Table 6 compares the equations of the line-of-best fit and the correlation coefficients.

\section{CACULATIONS}

\subsection{Equation of the Line of Best-Fit}

The equation of the line of best-fit was found from its slope and y-intercept. The slope was determined from the coordinates of two points on the line and the intercept, using the coordinates of a point in the equation $\mathrm{y}=\mathrm{mx}+\mathrm{b}$ and solving for $\mathrm{b}$. 
Miah M. Adel et al. / Journal of Social Sciences 9 (4): 164-172, 2013

Table 2. Probabilities for different sample sizes

\begin{tabular}{llll}
\hline Zodiacs & 100 & 200 & 300 \\
\hline Aries & 0.080 & 0.065 & 0.067 \\
Taurus & 0.070 & 0.070 & 0.057 \\
Gemini & 0.050 & 0.075 & 0.130 \\
Cancer & 0.060 & 0.090 & 0.070 \\
Leo & 0.100 & 0.095 & 0.067 \\
Virgo & 0.080 & 0.075 & 0.057 \\
Libra & 0.070 & 0.075 & 0.103 \\
Scorpio & 0.100 & 0.085 & 0.100 \\
Sagittarius & 0.070 & 0.065 & 0.053 \\
Capricorn & 0.100 & 0.095 & 0.083 \\
Aquarius & 0.140 & 0.130 & 0.150 \\
Pisces & 0.080 & 0.080 & 0.107 \\
& 1.000 & 1.000 & 1.000 \\
\hline
\end{tabular}

Table 3. Correlation coefficient for sample size 100

\begin{tabular}{|c|c|c|c|c|c|}
\hline Zodiac signs & $\mathrm{x}$ & $\mathrm{y}$ & xy & $\mathrm{x}^{2}$ & $\mathrm{y}^{2}$ \\
\hline Aries & 1 & 8 & 8 & 1 & 64 \\
\hline Taurus & & 7 & 14 & 4 & 49 \\
\hline Gemini & 3 & 5 & 15 & 9 & 25 \\
\hline Cancer & 4 & 6 & 24 & 16 & 36 \\
\hline Leo & 5 & 10 & 50 & 25 & 100 \\
\hline Virgo & 6 & 8 & 42 & 36 & 64 \\
\hline Libra & 7 & 7 & 49 & 49 & 49 \\
\hline Scorpio & 8 & 10 & 80 & 64 & 100 \\
\hline Sagittarius & 9 & 7 & 63 & 81 & 49 \\
\hline Capricorn & 10 & 10 & 100 & 100 & 100 \\
\hline Aquarius & 11 & 14 & 154 & 121 & 196 \\
\hline Pisces & 12 & 8 & 96 & 144 & 64 \\
\hline Sum of the cols. & 78 & 100 & 695 & 560 & 896 \\
\hline
\end{tabular}

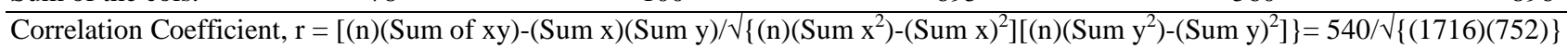
$=0.475$

Table 4. Correlation coefficient for sample size 200

\begin{tabular}{|c|c|c|c|c|c|}
\hline Zodiac signs & $\mathrm{x}$ & $\mathrm{y}$ & $\mathrm{xy}$ & $\mathrm{x}^{2}$ & $y^{2}$ \\
\hline Aries & 1 & 13 & 13 & 1 & 169 \\
\hline Taurus & 2 & 14 & 28 & 4 & 196 \\
\hline Gemini & 3 & 15 & 45 & 9 & 225 \\
\hline Cancer & 4 & 18 & 72 & 16 & 324 \\
\hline Leo & 5 & 19 & 95 & 25 & 361 \\
\hline Virgo & 6 & 15 & 90 & 36 & 225 \\
\hline Libra & 7 & 15 & 105 & 49 & 225 \\
\hline Scorpio & 8 & 17 & 136 & 64 & 289 \\
\hline Sagittarius & 9 & 13 & 117 & 81 & 169 \\
\hline Capricorn & 10 & 19 & 190 & 100 & 361 \\
\hline Aquarius & 11 & 26 & 286 & 121 & 626 \\
\hline Pisces & 12 & 16 & 192 & 144 & 256 \\
\hline Sum of the cols. & 78 & 200 & 1369 & 650 & 3478 \\
\hline
\end{tabular}


Table 5. Correlation coefficient for sample size 300

\begin{tabular}{|c|c|c|c|c|c|c|c|}
\hline Zodiac signs & $\mathrm{x}$ & $\mathrm{y}$ & & xy & $\mathrm{x}^{2}$ & & $y^{2}$ \\
\hline Aries & 1 & 20 & & 20 & 1 & & 400 \\
\hline Taurus & 2 & 17 & & 34 & 4 & & 289 \\
\hline Gemini & 3 & 26 & & 78 & 9 & & 676 \\
\hline Cancer & 4 & 21 & & 84 & 16 & & 441 \\
\hline Leo & 5 & 20 & & 100 & 25 & & 400 \\
\hline Virgo & 6 & 17 & & 102 & 36 & & 289 \\
\hline Libra & 7 & 31 & & 217 & 49 & & 961 \\
\hline Scorpio & 8 & 30 & & 240 & 64 & & 900 \\
\hline Sagittarius & 9 & 16 & & 144 & 81 & & 256 \\
\hline Capricorn & 10 & 25 & & 250 & 100 & & 625 \\
\hline Aquarius & 11 & 45 & & 495 & 121 & & 2025 \\
\hline Pisces & 12 & 32 & & 384 & 144 & & 1024 \\
\hline Sum of the cols. & 78 & 300 & & 2148 & 650 & & 8286 \\
\hline $\begin{array}{l}\text { Correlation Coe } \\
2376 / \sqrt{\{(1716)(94}\end{array}$ & $=[$ & xy)-(Sum & $\mathrm{x})(\mathrm{Sum}$ & $\left.\mathrm{y}) / \sqrt{\{(\mathrm{n})(\text { Sum }} \mathrm{x}^{2}\right)-($ Sum & $\left.\mathrm{x})^{2}\right][(\mathrm{n})(\mathrm{Sum}$ & $\left.y^{2}\right)-($ Sum & $\left.\left.\mathrm{y})^{2}\right]\right\}=$ \\
\hline \multicolumn{8}{|c|}{ Table 6. Comparison of results } \\
\hline Sample size & \multicolumn{3}{|c|}{ Best-line fit } & Correlation coefficient & \multicolumn{3}{|c|}{ Comments } \\
\hline 100 & & & \multicolumn{2}{|c|}{ moderately related } \\
\hline 200 & \multicolumn{3}{|c|}{$y=0.48 x+13.56$} & \multicolumn{2}{|l|}{0.48} & \\
\hline 300 & \multicolumn{3}{|c|}{$y=1.33 x+16.01$} & 0.59 & & \multicolumn{2}{|c|}{$\begin{array}{l}\text { moderately related } \\
\text { moderately related }\end{array}$} \\
\hline
\end{tabular}

\subsubsection{Sample Size of 100 Celebrities}

Sample size 100: $\mathrm{x}_{1}=2, \mathrm{y}_{1}=6.8, \mathrm{x}_{2}=9, \mathrm{y}_{2}=9.2$. Slope of the line, $\mathrm{m}=\left(\mathrm{y}_{2}-\mathrm{y}_{1}\right) /\left(\mathrm{x}_{2}-\mathrm{x}_{1}\right)=0.34$. Substitution of this value of the slope in equation $\mathrm{y}=\mathrm{mx}+\mathrm{b}$ and using $x=2$ and $y=6.8$, yields $b=6.12$. So, the equation of the line is: $y=0.34 x+6.12$.

\subsubsection{Sample Size of 100 Celebrities}

Sample size 200: $\mathrm{x}_{1}=3, \mathrm{y}_{1}=15, \mathrm{x}_{2}=7.2, \mathrm{y}_{2}=17$. Slope of the line, $\mathrm{m}=\left(\mathrm{y}_{2}-\mathrm{y}_{1}\right) /\left(\mathrm{x}_{2}-\mathrm{x}_{1}\right)=0.48$. Substitution of this value of the slope in equation $\mathrm{y}=\mathrm{mx}+\mathrm{b}$ and using $x=3$ and $y=15$, yields $b=13.56$. So, the equation of the line is: $y=0.48 x+13.56$.

\subsubsection{Sample Size of 100 Celebrities}

Sample size 300: $\mathrm{x}_{1}=3, \mathrm{y}_{1}=20, \mathrm{x}_{2}=6, \mathrm{y}_{2}=24$. Slope of the line, $\mathrm{m}=\left(\mathrm{y}_{2}-\mathrm{y}_{1}\right) /\left(\mathrm{x}_{2}-\mathrm{x}_{1}\right)=1.33$. Substitution of this value of the slope in equation $y=m x+b$ and using $x=3$ and $y=20$, yields $b=16.01$. So, the equation of the line is: $y=1.33 x+16.01$.

\subsection{Probabilities}

The probabilities of the occurrences of the zodiacs in a sample size were found by dividing the number of celebrities falling in a zodiac by the total number of celebrities in that sample. The probabilities of all samples have been tabulated in Table 2.

\subsection{Correlation Coefficients}

The correlation coefficient for each of the sample sizes was determined using the standard statistical procedure. The $x_{-}, y-$ and their derived values $x y-, x^{2}$ and $y^{2}$ - are tabulated in Table 3 to 5. Surprisingly, the two variables- $\mathrm{x}$ and $\mathrm{y}$ - show medium correlation.

\subsubsection{Correlation Coefficient for 100 Sample Size}

The quantities for the calculation of correlation coefficient have been tabulated in Table 3. The correlation coefficient for this sample size was 0.475 which is an indication of moderate correlation.

\subsubsection{Correlation Coefficient for 200 Sample Size}

The quantities required for the calculation of correlation for this sample size is tabulated in Table 4. The correlation coefficient for this sample size was 0.4797 which, too, is an indication of moderate correlation.

\subsubsection{Correlation Coefficient for 300 Sample Size}

The quantities required for the calculation of correlation for this sample size is tabulated in Table 5. The correlation coefficient for this sample size was 0.0.59 which is still an indication of moderate correlation. 


\section{RESULTS}

\subsection{Bar Plots}

Figure 2 to 4 shows the bar plots for 100, 200 and 300 sample sizes. It is observed that the number of celebrities in Aquarius outnumbers the same in all other zodiacs which have got fluctuating number of celebrities with the sample sizes.

The scatter plots for 100, 200 and 300 celebrities appear in Fig. 5 to 7, respectively. The line of best fit in them has the form $y=m x+b$ with different values of the slope $m$ and the $y$-intercept $b$.

The correlation coefficients for the 100, 200 and 300 sample sizes are found to be $0.48,0.48$ and 0.59 , respectively.

\subsubsection{Bar Plot for Sample Size 100}

The bar plot for the sample size of 100 is shown in Fig. 2. The zodiac Aquarius has, by far, the largest number of celebrities. The next ones are the Leo, the Scorpio and the Capricorn.

\subsubsection{Bar Plot for Sample Size 200}

The bar plot for the sample size of 200 is shown in Fig. 3. The zodiac Aquarius has still, by far, the largest number of celebrities. The next ones are the Leo and the Capricorn followed by the Cancer.

\subsubsection{Bar Plot for Sample Size 300}

The bar plot for the sample size of 300 is shown in Fig. 4. Again, the zodiac Aquarius has, by far, the largest number of celebrities. The next one is the Pisces followed by Libra and then by the Scorpio.

\subsection{Line of Best-Fit}

The standard statistical method was applied to find the line of best fit. The lines of best-fit through the scatter plots are shown in Fig. 5 to 7.

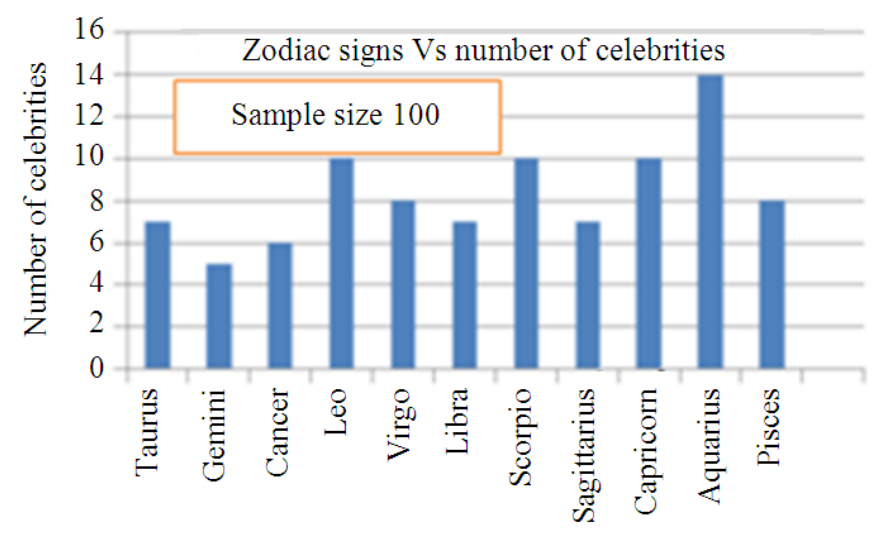

Fig. 2. Bar plot for 100 celebrities

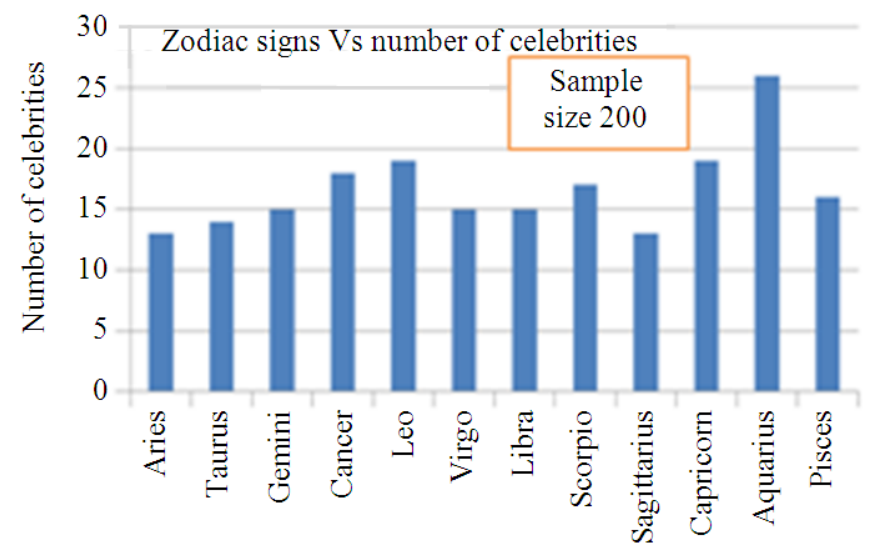

Fig. 3. Bar plot for 200 celebrities 
Miah M. Adel et al. / Journal of Social Sciences 9 (4): 164-172, 2013

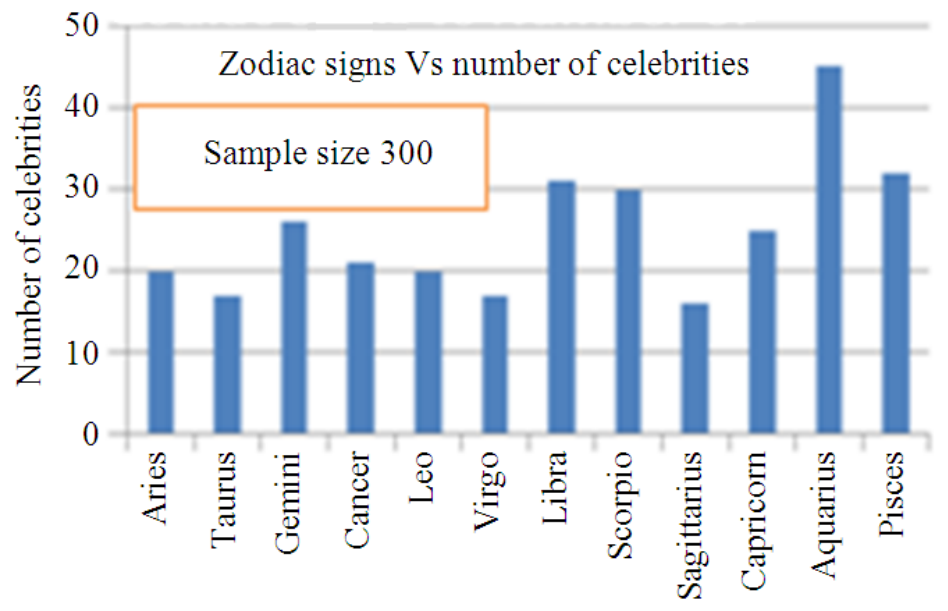

Fig. 4. Bar plot for 300 celebrities

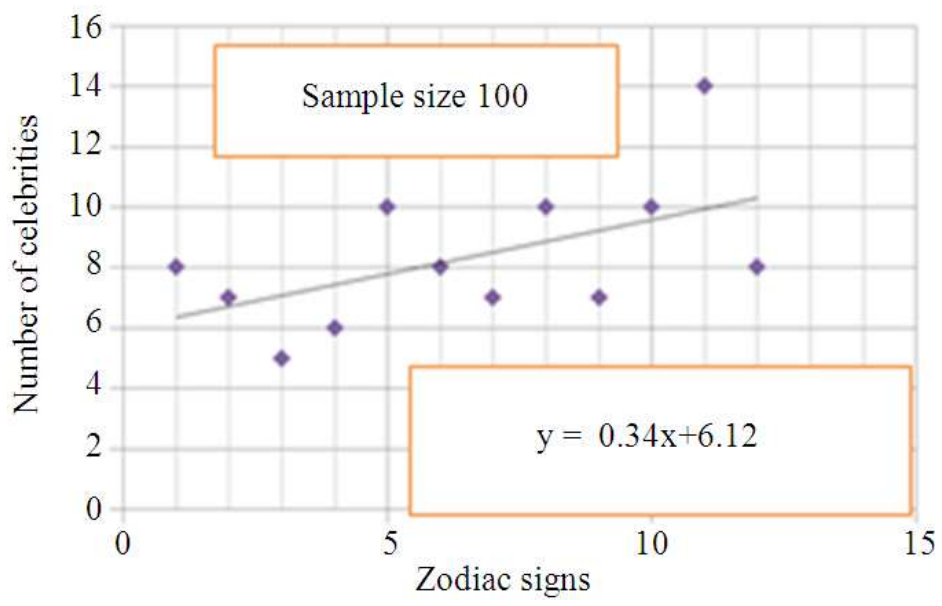

Fig. 5. Scatter plot for 100 celebrities

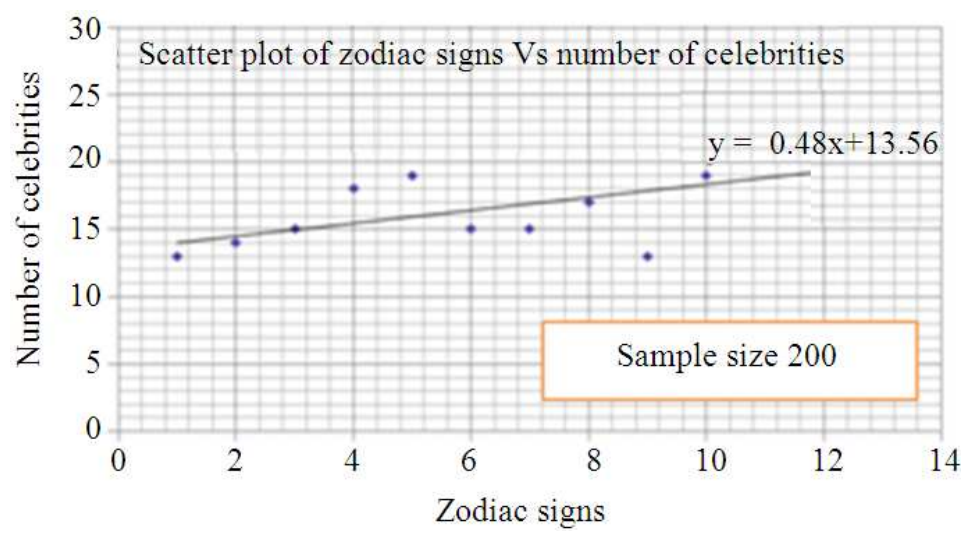

Fig. 6. Scatter plot for 200 celebrities 


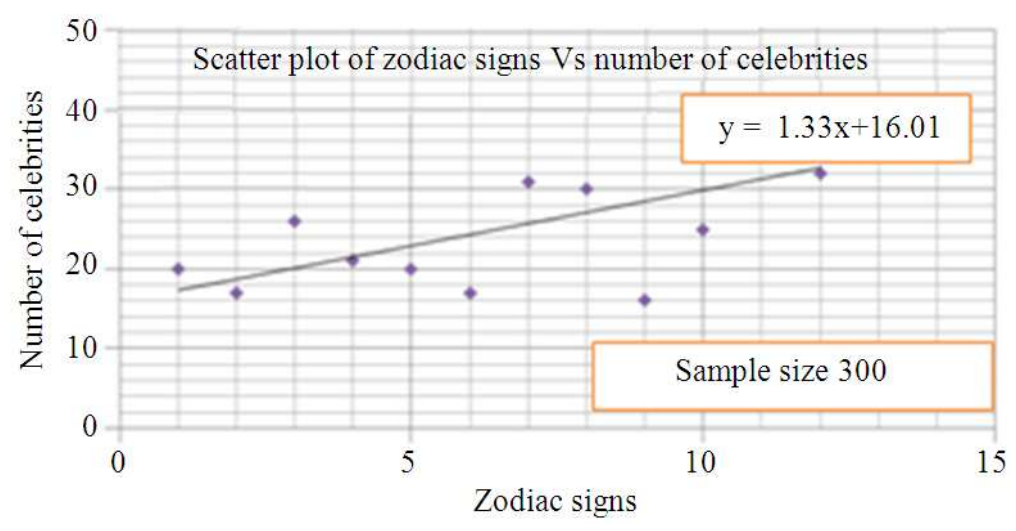

Fig. 7. Scatter plot of 300 celebrities

\subsubsection{Line of Best-Fit for Sample Size 100}

This is shown in Fig. 5. The equation of the line of best-fit is $\mathrm{y}=0.34 \mathrm{x}+6.12$

\subsubsection{Line of Best-Fit for Sample Size 200}

This is shown in Fig. 6. The equation of the line of best-fit is $\mathrm{y}=0.48 \mathrm{x}+13.56$

\subsubsection{Line of Best-Fit for Sample Size 300}

This is shown in Fig. 7. The equation of the line of best-fit is $\mathrm{y}=1.33 \mathrm{x}+16.01$

\section{COMPARISON OF RESULTS}

The results for the lines of best-fit and the correlation coefficients are shown in Table 6.

\section{DISCUSSION}

\subsection{Data Spread and Unbiasedness in Data Selection}

Samples of individuals of the current time or historical past who excelled in their respective professions nationally or internationally and whose personal information was accessible and whom media give the celebrity status were taken as the subjects of the study.

These individuals were among the academicians, discoverers, inventors, statesman, sportsmen, sportswomen, singers, actors, actresses, authors, social workers, etc. etc. recorded in history as well as in the present time. There are individuals in the samples from the time when movie stars and producers were not even borne and sports were not developed.
The random selection was not like the generation of random numbers. The names were picked without any choice of the place, time, national origin, sex, of the individuals.

The sample selection would be biased if the individuals were selected based on their dates of birth. The dates of birth were not known until the name was picked up and there was no rejection or preference of any individuals for being born under some zodiacs.

Setting some conditions or criteria for selection of the sample elements, brings some restrictions to the study. Leaving it open makes the result a more general one out of diversity of age, sex, ethnicity etc.

\subsection{Limitation of the Study}

It was not possible to study age-wise because individuals might have multiple achievements. It would put some uncertainty as to the age of his/her achieving celebrity status. A sex-wise study would not be informative because of the number of women celebrity would be a few. Even in the sixties, Harvard University was not admitting female students until the early sixties (Dr. Vera Rubin's lecture at the First Vatican Summer School in Observational Astronomy and Astrophysics, 1986, when the principal author was a student). If there is such a blockage as this, the possibility of rising to celebrity status would be stopped. Also, an ethnicity-based study will not be worthwhile because of the majority of the African-Americans are yet to be the first generation college-going ones and a few celebrities are in this ethnic group.

\subsection{Sample Size Variation Objectives}

The variation of the sample size was made to find if it was a general result independent of the sample of 
individuals or dependent on any particular sample of individuals. Even though the first selected sample of 100 individuals showed the trend reported in the article, if there was any otherwise effect, it would be reflected with the addition of 100 more and later 200 more individuals in the samples. Further, increasing the sample size in steps should avoid the requirement of a very large number individuals in the sample because of the reflection of the same trends in all the samples.

\subsection{Academic Values of the Article}

This article has research as well as academic values. The research value lies in its new result. Its academic values relate to the coauthors who are high school juniors and who learn the simple statistics. Mathematics textbooks give examples of scatter plots, least-squares-fit, correlation, etc. Some math textbook author may illustrate these statistical principles with the help of these data points. Three different sample sizes were selected to see if the trend remains the same. This was a part of the procedure in scientific investigations and was followed in the interest of teaching steps in scientific investigation to junior investigators.

\subsection{Arbitrarily Zodiacs Numbering}

In making the plots, Aries was arbitrarily assigned the number " 1 ". Even if Aries is not marked by " 1 " by another data analyzer, neither the result nor the academic value changes as long as the consistency is maintained in all cases of assigning the numbers to the zodiacs.

\subsection{Prohibition to Study for the Public}

The category of subjects could not be extended beyond the celebrities to common people to make a general study because the law makes people's vital records inaccessible. The gives us a good picture of the favored zodiac sign, the Aquarius, for celebrities. The principal author approached the state office of vital records and the CDC, no common person's birth records were made available for this research purpose.

\subsection{Indeterminate Roles of Zodiacs Traits}

Each of the zodiac signs has several traits. It was not possible to see if all the individuals in a zodiac sign showed all or some of the traits of the sign. Also, it was not possible to see if any particular traits made them celebrities.

\subsection{Further Academic Value}

The theoretical prediction or the best-fit line cannot be used for any $\mathrm{x}$ beyond $1 \leq \mathrm{x} \leq 12$. It was in the interest of learning practical lessons of algebra for the juniors. Someday some illustrated algebra textbook can use these relations in function evaluation problems. The probability calculation, determination of the correlation coefficients, scatter plots and the line of best fits were also in the interest of the junior investigators' learning of practical lessons in probability statics and algebra and in the analysis data in research. The results open up a new area of research if common people's vital records were made available for extended research. Astrological beliefs could be tested with practical data.

\section{CONCLUSION}

The highest number of celebrities is born in the zodiac sign Aquarius. All other zodiacs have variations in the number of celebrities with the increase of sample sizes. The best-line fit in the scatter plot of the data shows that the number of celebrity births increases linearly as we go down the list of the zodiac signs. Zodiac signs and the number of celebrities are moderately related as shown by the correlation coefficients in the range of 0.50 to 0.60 . The result and the procedure used have both the research and academic values.

\section{ACKNOWEDGEMENT}

The authors are sincerely grateful to the individuals and/or agencies whose information has been used in the preparation of this article.

\section{REFERENCES}

http://www.astrology.com.au/astrology/12-signs-of-thezodiac/www.wikepedia.org

http://www.statisticshowto.com/articles/how-tocompute-pearsons-correlation-coefficients/ www.psychicguild.com 